\title{
Efficacy of antibiotics and non-steroidal anti-inflammatory drugs in non-complicated acute bronchitis
}

\author{
Elisa Maria Fiorelli • Laura Ferrari • \\ GrAM (Gruppo di Autoformazione Metodologica)
}

Received: 13 June 2014 / Accepted: 23 June 2014/Published online: 11 July 2014

(C) SIMI 2014

\section{Background}

Acute bronchitis is defined as an acute self-limiting respiratory tract infection, occurring in a patient without chronic lung disease, in which cough, which may or may not be productive, is the predominant feature. The process affects $5 \%$ of adults annually, and it accounts for a large number of the infections attended by family physicians [1]. Acute bronchitis is mainly a viral infection, and the role of bacteria in this condition continues to be controversial. Despite the limited evidence to support the use of antibiotics for acute bronchitis, the majority of patients are still treated with antibiotics [2]. Little evidence is available about the role of non-steroidal anti-inflammatory drugs, antitussive agents or other over-the-counter medicines.

\section{Summary}

Llor et al. [3] conducted a single-blinded, placebo-controlled study comparing the efficacy of ibuprofen and amoxicillin-clavulanic acid with placebo in reducing the number of days with frequent cough in a group of adults with acute bronchitis and discoloured sputum without associated respiratory comorbidity or immunosuppression. Patients were recruited by 25 general practitioners from 9 primary care centres in Catalonia. To be eligible, patients needed to present symptoms associated with respiratory tract infection of less than one week's duration (cough and discoloured sputum, and at least one other criterion such as dyspnoea, wheezing, chest discomfort, or chest pain). Main exclusion

E. M. Fiorelli $(\square) \cdot$ L. Ferrari

Medicina III, Ospedale San Paolo, Milan, Italy

e-mail: elisafiorelli@live.it criteria were antibiotic, anti-inflammatory drugs, or corticosteroid use in the previous 2 weeks; radiological signs of pneumonia; signs of severe infection, history of gastrointestinal haemorrhage or intolerance to anti-inflammatory treatment; associated comorbidity; emergency situation; and living in residential care. Patients were randomised to receive either ibuprofen ( $600 \mathrm{mg} 3$ times daily for 10 days, 136 patients) or amoxicillin-clavulanic acid (500 mg/ $125 \mathrm{mg} 3$ times daily for 10 days, 137 patients) or placebo (3 times daily for 10 days, 143 patients). Patients follow-up consisted of 3 control visits: inclusion visit (clinical examination and $\mathrm{C}$ reactive protein rapid test in capillary blood); first follow-up visit (after 2-4 days, evaluation of the clinical course of symptoms); and second follow-up visit (after 11-13 days), in which patients were classified as cured, improved or failure. Patients' symptoms were evaluated by a symptom diary that considered five items: disease severity, daytime cough, nighttime cough, limitation in daily activities, and febrile sensation. The primary outcome was the number of days with frequent cough in the intention to treat population. Secondary outcomes were clinical success (defined as cured or improvement) at the end of the second follow-up visit and the time of resolution of the total symptom scores. As regards primary outcome, no statistically significant differences were observed between the study groups (ibuprofen group, 9 days (95\% CI 8-10); amoxicillin-clavulanic acid group, 11 days (95\% CI $10-12)$; placebo group, 11 days (95\% CI 8-14), $p=0.25$ ). No statistically significant differences were found between the three arms in the secondary outcome. Patients treated with antibiotics had a higher percentage of adverse events (12\% in the amoxicillin-clavulanic group, $5 \%$ in the ibuprofen group and $3 \%$ in the placebo one. $p=0.008$ ); adverse events were mostly mild, except one case of digestive haemorrhage in the antibiotic arm. 


\section{Strengths of the study}

- The study is methodologically well-conducted despite being a single-blind study.

- It deals with a clinically relevant problem; in view of the high prevalence of the disease and the extensive use of the antibiotic therapy, appears to be very relevant in primary care settings.

\section{Weaknesses of the study}

- There was a violation of the study protocol with respect to the inclusion criteria. In the study, protocol was mentioned to include patients with presence of symptoms of at least 1 week of duration [4].

\section{Question marks}

- The primary outcome was the number of days with frequent cough; it was a subjective outcome, based on information collected from symptom diaries. This method has been previously validated, and the feasibility of other methods of symptoms evaluation would have been difficult in a primary care setting. However, as the authors said there could be some problem in compiling the diary, especially with older patients. We wonder if a more objective outcome, such as the days of fever, would have been more appropriate.

- The authors used a block randomisation method, and they clearly explain the random allocation sequence generation; however, the allocation concealment method is less inferable.

- The authors did not consider macrolides to avoid the possibility of clinical improvement due to an antiinflammatory effect; however, macrolides are frequently used as first-choice antibiotics in acute bronchitis of a suspected bacterial cause as they seem to be more effective than amoxicillin or amoxicillin-clavulanic acid [5].

- We wonder why the authors have chosen a lower dose of amoxicillin-clavulanic acid $(500 \mathrm{mg} / 125 \mathrm{mg}, 3$ times daily) instead of the most commonly used dose of $875 \mathrm{mg} / 125 \mathrm{mg}, 3$ times daily.

\section{Sponsorship}

This clinical trial was supported by a grant from the Instituto de Salud Carlos III (Spanish Ministry of Health) (EC07/90333). The funder had no role in the design, conduct, or analysis of this study.

\section{Clinical bottom line}

There is no evidence that therapy with amoxicillin-clavulanic acid or non-steroidal anti-inflammatory drugs is better than placebo to improve symptoms of acute bronchitis in patients without associated respiratory comorbidity or immunosuppression; purulent sputum should not be considered a criterion to start an antibiotic therapy.

\section{Conflict of interest None.}

\section{References}

1. Woodhead M, Blasi F, Ewig S et al (2011) Guidelines for the management of adult lower respiratory tract infections-full version. Clin Microbiol Infect 17(Suppl 6):E1-E59. doi:10.1111/ j.1469-0691.2011.03672.x

2. Smucny J, Fahey T, Becker L et al (2004) Antibiotics for acute bronchitis. Cochrane Database Syst Rev 18(4):CD000245

3. Llor C, Moragas A, Bayona C et al (2013) Efficacy of antiinflammatory or antibiotic treatment in patients with non-complicated acute bronchitis and discoloured sputum: randomised placebo controlled trial. BMJ 4(347):f5762. doi:10.1136/bmj. f5762

4. Llor C, Moragas A, Bayona C et al (2011) Effectiveness of antiinflammatory treatment versus antibiotic therapy and placebo for patients with non-complicated acute bronchitis with purulent sputum. The BAAP Study protocol. BMC Pulm Med 21(11):38. doi:10.1186/1471-2466-11-38

5. Panpanich R, Lerttrakarnnon P, Laopaiboon M (2008) Azithromycin for acute lower respiratory tract infections. Cochrane Database Syst Rev 23(1):CD001954. doi:10.1002/14651858 (CD001954.pub3) 